\title{
Is an Accelerating Robot Perceived as Energetic or as Gaining in Speed?
}

\author{
Matthijs L. Noordzij \\ Martin Schmettow \\ Melle R. Lorijn \\ Cognitive Psychology and Ergonomics, University of Twente, Drienerlolaan 5, 7522NB Enschede, The Netherlands \\ +31534892589 \\ +31534893874 \\ m.I.noordzij@utwente.nl \\ m.schmettow@utwente.nl \\ mrlorijn@gmail.com
}

\begin{abstract}
Previous studies have found that basic movement characteristics of a robot influence the emotional attributes people perceive independent of the embodiment of the motion (e.g. iCat vs. Roomba). Here, with a very simple LEGO robot, we replicate these associations between levels of acceleration and curvature and the extent to which positive and negative emotions are attributed. Importantly, we also show that these associations might not be valid. Prior to the emotional questionnaires participants were asked neutral questions on what they deemed relevant observations pertaining to the different robot motions. Only $3 \%$ of the remarks coincided with the emotional terms found in the questionnaires. HRI researchers interested in what people attribute to robot motion should be mindful of participant heuristics and experimenter biases. We provide some suggestions how to create experiments that are robust against these biases.
\end{abstract}

\section{Categories and Subject Descriptors}

H.1.2 User/Machine Systems: Human factors

\section{General Terms}

Measurement, Reliability, Experimentation, Human Factors.

\section{Keywords}

Robot Motion, Emotion, PANAS, Attribution Heuristic, Response Anchoring.

\section{INTRODUCTION}

In the field of Human-Robot Interaction (HRI) there have been efforts to find generalizable rules governing human (emotional) reactions to certain variations in robot behavior. For example, it has been suggested that motion characteristics of a robot can influence the attribution of affect in a reliable way, independent of the appearance of the robot and the particular way the motion is embodied [5]. In this latter research curvature and acceleration parameters were varied in two robot platforms: an iCat, which is a robotic cat-head that can pan and tilt, and a Roomba, which is a disc-like robotic vacuum cleaner that can drive around. Therefore, the three levels of curvature and acceleration were instantiated in very different ways in these two embodiments (e.g. tilting the head faster vs. moving faster on the floor). Yet, the researchers found consistent patterns in how people judged the emotional

Permission to make digital or hard copies of part or all of this work for personal or classroom use is granted without fee provided that copies are not made or distributed for profit or commercial advantage, and that copies bear this notice and the full citation on the first page. Copyrights for third-party components of this work must be honored. For all other uses, contact the owner/author(s).

Copyright is held by the author/owner(s).

HRI'14, March 3-6, 2014, Bielefeld, Germany.

ACM 978-1-4503-2658-2/14/03.

http://dx.doi.org/10.1145/2559636.2559793 attributes of the two robots (as judged by subjective scales) as a function of motion characteristics. These kinds of findings promise both a more generalizable scientific framework for HRI and direct design guidelines.

Here, we try to replicate part of this research on motion characteristics with yet another robot: a LEGO Mindstorms cart. If the claim that the relation between emotional attribution and motion parameters is independent of embodiment we should be able to find the same associations with a simple robot toy. In addition, we independently assess whether the choices people make on questionnaires probing emotional attributes of the robot correspond to what people spontaneously associate with the variations in robot motion. In [5] people did also spontaneously give (emotional) comments on the robot, but this always happened while they were filling in questionnaires consisting of only emotional terms. In addition, a semi-structured interview was done, but only after people had seen (and committed choices to) the emotional questionnaires. To address the possible confound of priming participants with emotional terms, we asked people to answer to open questions on the robot before having seen, let alone answered on, emotional questionnaires.

\section{METHOD}

\subsection{Participants}

We recruited 28 participants ( 8 female), aged between 18 and 27 years.

\subsection{Materials}

The robot we used was the starter "cart" model of the Lego Mindstorms ${ }^{\circledR} \quad$ NXT 2.0 set (a small tracked vehicle of approximately $20 \times 20 \times 20 \mathrm{~cm}$.) with an ultrasonic sensor at the front. From the set of questionnaire used in [5] we selected the PANAS [8], which measures positive and negative affect by letting people rate the appropriateness (from 1 to 5) of 10 positive and 10 negative emotional terms. We also used a simple open question form in which participants were asked to fill in the responses they deemed relevant after each robot motion. We tried to emulate the values used in [5] for curvature and acceleration as good as possible on the Lego robot. For curvature we could closely copy the original (Roomba) values by letting the robot drive a path with a mean curvature $(=1 /$ radius of the circle $)$ of .85 (low curvature), 1.5 (medium curvature) and 2.2 (high curvature) meters. For acceleration we ended up with a rough, relative approximation, because the absolute acceleration was much slower of the LEGO robot than of the Roomba. We programmed the robot to increase its motor power by $10 \%$ per time unit. The different time units (in seconds) were 3.5 (low acceleration), 1.05 (medium acceleration), and .57 (high acceleration). 


\subsection{Design and procedure}

Before the start of the experiment participants filled in an informed consent form (the study was approved by the local ethics committee). The participant watched the nine movement patterns (each a combination of a level of acceleration and curvature). The patterns were quasi-randomized (5 different orders) over participants, and after each pattern participants were asked to write down what their response was to what they had just seen. It was stressed that they were completely free in writing down what they found relevant. Subsequently, the nine patterns were shown again, each time followed by the PANAS.

\section{RESULTS}

\subsection{Emotional questionnaire}

The absolute averages on the PA $(23.7$, standard deviation $(\mathrm{sd})=$ 1.6) and NA $(16.9, \mathrm{sd}=1.0)$ for our Lego robot and those for the Roomba [5] $(\mathrm{PA}=23.0, \mathrm{sd}=1.5$; NA 17.1, $\mathrm{sd}=1.2)$ ) were strikingly similar. In addition, the repeated measures ANOVA with curvature (low, medium, high) and acceleration (low, medium, high) revealed similar main and interaction effects. As in [5] by far the strongest effect on the PANAS was the main effect of acceleration for PA $\left(\mathrm{F}(2,54)=14.4, \mathrm{p}<.001, \eta^{2}=.35\right)$, indicating increasing positive affect with increasing acceleration.

\subsection{Open questions}

The qualitative data was analyzed with constant comparison analysis and classical content analysis [3], resulting in 299 coded utterances. These were categorized into six categories: Description of motion (63\% of the utterances), Anthropomorphic (27\%), Emotional terms (3\%), Irrelevant/ question (3\%), Mechanical (2\%), Comparison to other vehicles $(1 \%)$. Importantly, only a very small number of utterances $(3 \%)$ contained emotional attributes similar to the ones that are used in questionnaires such as the PANAS. Mostly, people simply described the motion (i.e. "the robot is accelerating faster than the previous time"). The answers to the open questions in our study seem to suggest that the emotional dimensions in the PANAS questionnaire were not representative of what people actually associated with the movement changes.

\section{DISCUSSION AND CONCLUSION}

People attribute complex emotions and social behavior to moving objects, even when they are just simple geometrical shapes [1]. Therefore, it is tempting to take this association and assume that it is always valid to investigate the emotional attributions motion variations will evoke. Establishing how changes in robot motion (independent of embodiment) steer emotional attribution would have enormous benefits. However, here we show that strictly separating a neutral experimental phase (i.e. "What do people associate with robot motion?") from an emotional phase ("What emotion do people associate with robot motion?") calls into question the validity of only asking questions about the emotions people attribute to robot motion. We suggest that over these different robotic embodiments people are highly reliably mapping clearly visible differences in motion characteristics to the only other dimension that is available in the form of an emotional questionnaire.

It has long been known that respondents in closed question surveys can limit themselves with ease to the predefined response categories, even when these are incomplete [7]. For example, it has become known as the attribute substitution heuristic when people judge on irrelevant attributes when relevant information is not readily available to them [2]. Hence, in the case of [5] the possibility cannot be ruled out that participants responded to the closed questions in emotional terms because they were forced to. In the subsequent open questions they could have responded congruently, because of attribute substitution, or because they guessed what the researchers were after. Furthermore, response anchoring [4] could explain why the observed effects appeared so uniform, independent of the embodiment.

For drawing conclusions on the crucial question of spontaneous associations with robot motion, further research has to clarify whether movement patterns generally and primarily evoke spontaneous emotional associations, and how intense these associations are by absolute means. Implicit experimental techniques, such as the Stroop task can serve to non-reactively assess spontaneous associations [6]. Potential anchoring effects could be unrevealed by studying human reactions to two levels of robot acceleration in a narrow range (acceleration range is not much wider than the two levels) or wide range (acceleration range is much greater than the two levels, see [4]). Finally, psychophysiological measures (e.g. electrodermal activity) could provide absolute measures of arousal.

\section{REFERENCES}

[1] Heider, F. and Simmel, M. An Experimental Study of Apparent Behavior. The Am. J. of Psy. 57, 2 (1944), 243259. DOI= http://dx.doi.org/10.2307/1416950.

[2] Kahneman, D. and Frederick, S. Representativeness revisited: Attribute substitution in intuitive judgment. In T. Gilovich, D. Griffin and D. Kahneman, eds., Heuristics of Intuitive Judgment: Extensions and Applications. Cambridge University Press, New York, 2002.

[3] Leech, N.L. and Onwuegbuzie, A.J. An array of qualitative data analysis tools: A call for data analysis triangulation. School Psy. Q. 22, 4 (2007), 557-584. DOI= http://dx.doi.org/10.1037/1045-3830.22.4.557.

[4] Lynch, J.G., Chakravarti, D., and Mitra, A. Contrast Effects in Consumer Judgments : Changes in Mental Representations or in the Anchoring of Rating Scales? J. of Cons. Res., 18, 3 (2013), 284-297.

[5] Saerbeck, M. and Bartneck, C. Perception of affect elicited by robot motion. Proceeding of the 5th ACM/IEEE international conference on Human-robot interaction-HRI '10, ACM Press (2010), 53-60. DOI= http://dx.doi.org/10.1109/HRI.2010.5453269.

[6] Schmettow, M., Noordzij, M.L., and Mundt, M. An implicit test of UX: Individuals Differ in What They Associate with Computers. CHI '13 Extended Abstracts on Human Factors in Computing Systems on - CHI EA '13, ACM Press (2013), 2039-2048. DOI= http://dx.doi.org/10.1145/2468356.2468722.

[7] Schuman, H. and Presser, S. The Open and Closed Question. Am. Socio. Rev., 44, 5 (1979), 692-712. DOI= http://dx.doi.org/10.2307/2094521.

[8] Watson, D., Clark, L. and Tellegen, A. Development and validation of brief measures of positive and negative affect: the PANAS scales. J. of Pers. and Soc. Psy. 54, 6 (1988), 1063-70. DOI= http://dx.doi.org/10.1037/00223514.54.6.1063. 